\title{
Different clinical presentation in a patient with two novel pathogenic variants of the FBXL4 gene
}

\author{
Engin Köse ${ }^{1 \oplus}$, Melis Köse ${ }^{2 \oplus}$, Selvinaz Edizer ${ }^{3 \oplus}$, Zeynep Akışın ${ }^{4}$, \\ Zehra Burcu Yilmaz ${ }^{5}$, Ahmet Şahin ${ }^{5 \oplus}$, Ferah Genel ${ }^{6 \odot}$ \\ ${ }^{1}$ Division of Pediatric Metabolism and Nutrition, Department of Pediatrics, Ankara University Faculty of Medicine, Ankara; ${ }^{2}$ Division \\ of Pediatric Metabolism and Nutrition, Department of Pediatrics, Katip Çelebi University Faculty of Medicine, İzmir; Divisions of \\ ${ }^{3}$ Pediatric Neurology and ${ }^{6}$ Allergy and Immunology, ${ }^{5}$ Department of Pediatrics, ${ }^{4}$ Nutrition and Dietetics, Dr. Behçet Uz Children \\ Research and Training Hospital, Izmir, Turkey.
}

\begin{abstract}
Background. The recently described FBXL4-related encephalomyopathic mitochondrial DNA depletion syndrome 13 (MTDPS13) manifests with severe encephalopathy, early-onset lactic acidosis, hypotonia, developmental delay and feeding difficulty. Less than 100 cases with FBXL4-related MTDPS13 and 47 pathogenic mutations in the FBXL4 gene have been identified thus far. Here, we describe a patient diagnosed with MTDPS13 with two novel variants of the FBXL4 gene.

Case. A 51-day-old male was admitted with the complaint of bloody stool. His physical examination revealed facial dysmorphic features, developmental delay and truncal hypotonia with lack of head control. Laboratory investigations showed anemia, neutropenia, metabolic acidosis with hyperlactatemia, elevated fumaric acid, 2-ketoglutaric acid in urine and elevated alanine level in plasma which were consistent with mitochondrial dysfunction. Brain magnetic resonance imaging (MRI) showed large ventricles, thin corpus callosum and poor myelination. Drug-resistant epilepsy developed during the clinical follow-up. Ketogenic diet was initiated for intractable epilepsy; which was then interrupted due to severe metabolic acidosis. Compound heterozygous pathogenic variants were detected in the FBXL4 gene [p.Gly258* (c.772G>T, Exon 5)/p.Trp354Ser (c.1061G>C, Exon 6)] with whole-exome sequencing.

Conclusion. We detected two novel variants of the FBXL4 gene. To the best of our knowledge, this is the first case in the literature that presented with gastrointestinal bleeding as an encephalomyopathic form of mitochondrial DNA depletion syndromes and for whom ketogenic diet was initiated due to intractable epilepsy, which was not reported in previous cases.
\end{abstract}

Key words: FBXL4, MTDPS13, novel variants.

The FBXL4 (F-box and leucine-rich repeatcontaining protein) related encephalopathy referred to as encephalomyopathic mitochondrial DNA depletion syndrome 13 (MTDPS13) was first described in 2013. ${ }^{1}$ The FBXL4 mutation has pathogenetic effects on mitochondrial respiratory chain enzyme activities, disturbs the dynamic mitochondrial network and causes loss of mitochondrial

$凶$ Engin Köse

enginkose85@hotmail.com

Received 26th July 2019, revised 3rd December 2019, accepted 9th January 2020. membrane potential as well as mtDNA depletion. ${ }^{1}$ Most patients with FBXL4-related MTDPS13 present with severe encephalopathy, early-onset lactic acidosis, hypotonia, developmental delay and feeding difficulty. ${ }^{2-4}$ Cardiac, ophthalmological and genitourinary manifestations, seizure and hyperammonemia are reported as the less common features of the condition. ${ }^{4}$

To date, 94 patients with FBXL4-related MTDPS13 have been reported from different countries. Forty-seven pathogenic mutations in FBXL4 have been identified, and no genotypephenotype correlation has been determined. ${ }^{5}$ 
Here, we describe the $95^{\text {th }}$ patient with FBXL4-related MTDBS13 who presented with intestinal dysmotility, hypotonia, lactic acidosis and refractory epilepsy and had two novel pathogenic variants of the FBXL4 gene. To the best of our knowledge, this is the second patient with FBXL4-related MTDBS13 for whom ketogenic diet treatment was provided.

\section{Case Report}

A 51-day-old male was admitted to emergency department with the complaint of bloody stool. He was born to nonconsanguineous parents after a full-term uneventful pregnancy. His birth weight, height and head circumference were $2680 \mathrm{~g}$ (-1.6 SD), $50.5 \mathrm{~cm}(0.23 \mathrm{SD})$ and 35.5 $\mathrm{cm}(0.43 \mathrm{SD})$, respectively. In postnatal history, treatment due to a diagnosis of pneumonia for one month was reported by his parents. He had been exclusively breast-fed until the age of one month and his mother then proceeded feeding the infant with commercial formula. There was no family history of genetic disease or congenital abnormalities. The patient had a 3-year-old healthy brother.

During the physical examination, facial dysmorphic features such as micrognathia, low-set ear, bossing forehead and long philtrum were noted (Fig. 1). His weight was $4000 \mathrm{~g}$ (-1.53 SD), height was $55 \mathrm{~cm}$ (-0.61SD) and head circumference was $39.5 \mathrm{~cm}(0.26 \mathrm{SD})$. Developmental delay and truncal hypotonia with lack of head control were observed. While abdominal distension was detected, there was no hepatomegaly or splenomegaly. Ophthalmological evaluation revealed optic atrophy. Hearing impairment was not detected. Other systemic examinations were unremarkable.

Laboratory investigations showed anemia (hemoglobin $7.6 \mathrm{mg} / \mathrm{dl})$, neutropenia $\left(0.8 / 10^{3} /\right.$ $\mathrm{uL}$ ), metabolic acidosis ( $\mathrm{pH}: 7.22, \mathrm{HCO}_{3}: 14.5$ $\mathrm{mmol} / \mathrm{L}, \mathrm{pCO}_{2}: 20 \mathrm{mmHg}$ ) with hyperlactatemia (101 mmol/L, RR: <2.2). Renal function tests and transaminase levels were within normal range. Serum ammonia level was normal. Metabolic screening revealed elevated fumaric acid ( $45 \mu \mathrm{mol} / \mathrm{L}$; RR: 0 - 28) and 2-ketoglutaric acid (2743 $\mu \mathrm{mol} / \mathrm{L}$; RR: 0 - 631) in urine, and elevated alanine (580 $\mu \mathrm{mol} / \mathrm{L}$; RR: 139 - 474) level in plasma which were consistent with mitochondrial dysfunction. Acylcarnitine profile was nonspecific. For the evaluation of etiology of gastrointestinal tract bleeding, specific Ig E levels and skin prick test were assessed. No evidence was revealed for $\operatorname{IgE}$ mediated food allergies.

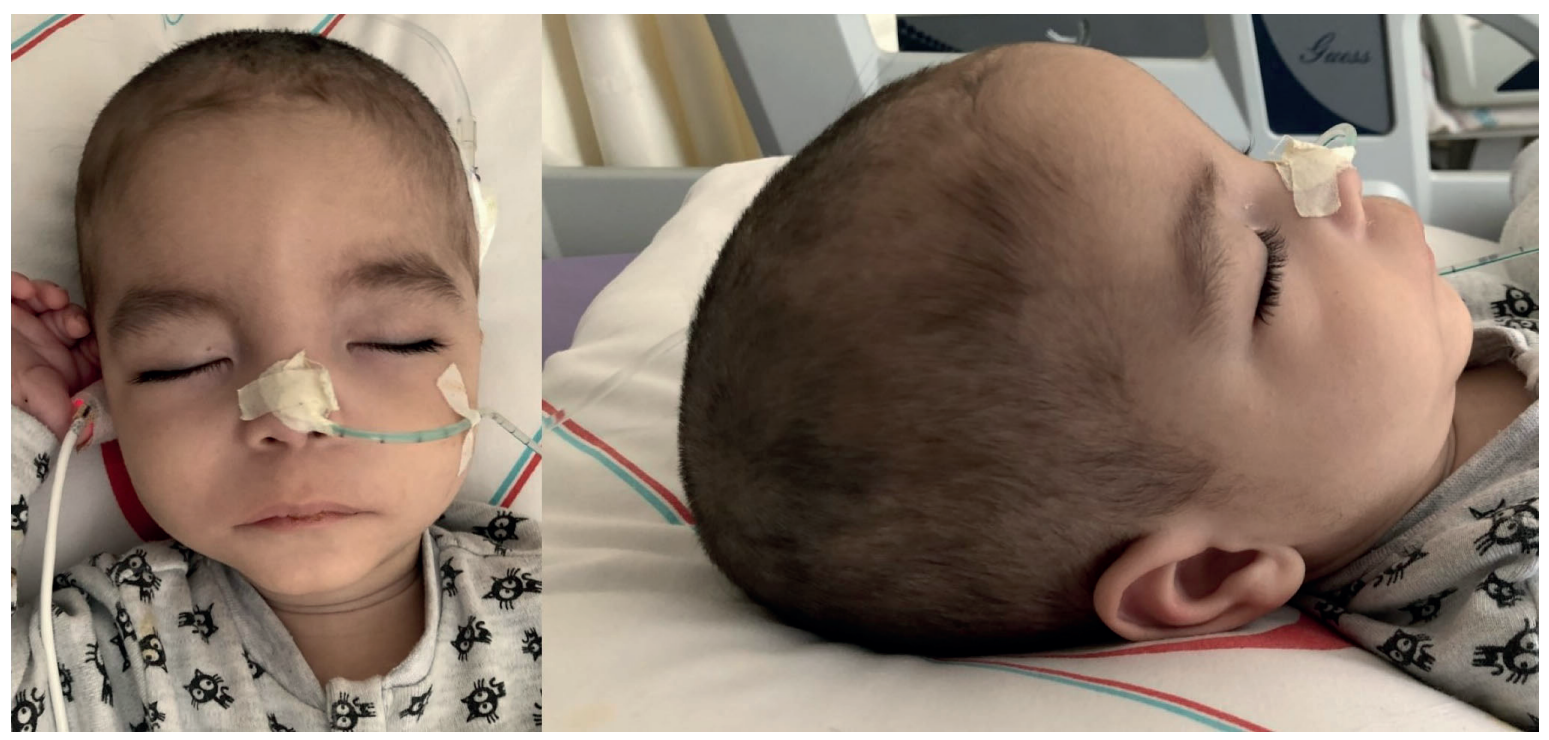

Fig. 1. Facial dysmorphic features of the patient. 
Abdominal sonography, echocardiogram, electrocardiogram and electroencephalogram (EEG) were normal. Brain magnetic resonance imaging (MRI) showed large ventricles and thin corpus callosum (Fig. 2a). Lactate peak was detected in brain MR spectroscopy (MRS) (Fig. 2b). Abdominal X-ray (posterior-anterior view) revealed dilated bowel (Fig. 2c). Clinical state of patient did not allow us to preform colonoscopy.

Metabolic acidosis and hyperlactatemia were treated with sodium bicarbonate and dichloroacetic acid. Coenzyme Q10, riboflavin, thiamine, biotin treatments were initiated for the differential diagnosis of mitochondrial disease. Nasogastric tube feeding was required due to hypotonia, dysphagia and feeding difficulties. Treatment with domperidone and ranitidine was initiated upon the findings of gastrointestinal dysmotility. Elimination diet with amino-acid based infant formula was initiated. However, episodic lower GI bleedings were observed and non-IgE mediated food allergies were ruled out. During his follow-up with these treatments, compound heterozygous pathogenic variants were detected in the FBXL4 gene [p.Gly258* (c.772G>T, Exon 5) / p.Trp354Ser (c.1061G>C, Exon 6)] with wholeexome sequencing. Genetic investigation was performed for his parents and sibling (Fig. 2d).

After 7 months, when he was 9 months old, the patient presented with feeding difficulty and fever. Pneumonia was diagnosed. Nystagmus, roving eyes and jerks of left arm were noted during the clinical examination, and EEG showed slow, irregular background activity and asynchronous sharp waves activity consistent with myoclonic epileptic encephalopathy (Fig. 2e). Brain MRI revealed large ventricles in addition to cerebral and cerebellar atrophy (Fig. 2f). Treatment with phenobarbital, levetiracetam and vigabatrin was initiated due to intractable epilepsy and ketogenic diet was introduced. However, while blood ketone level reached $5 \mathrm{mmol} / \mathrm{L}$ on the fifth day of the ketogenic diet, metabolic acidosis and hyperlactatemia occurred. Ketogenic diet was therefore discontinued. Finally, epileptic activity was treated with phenobarbital, levetiracetam, vigabatrin and topiramate medications.
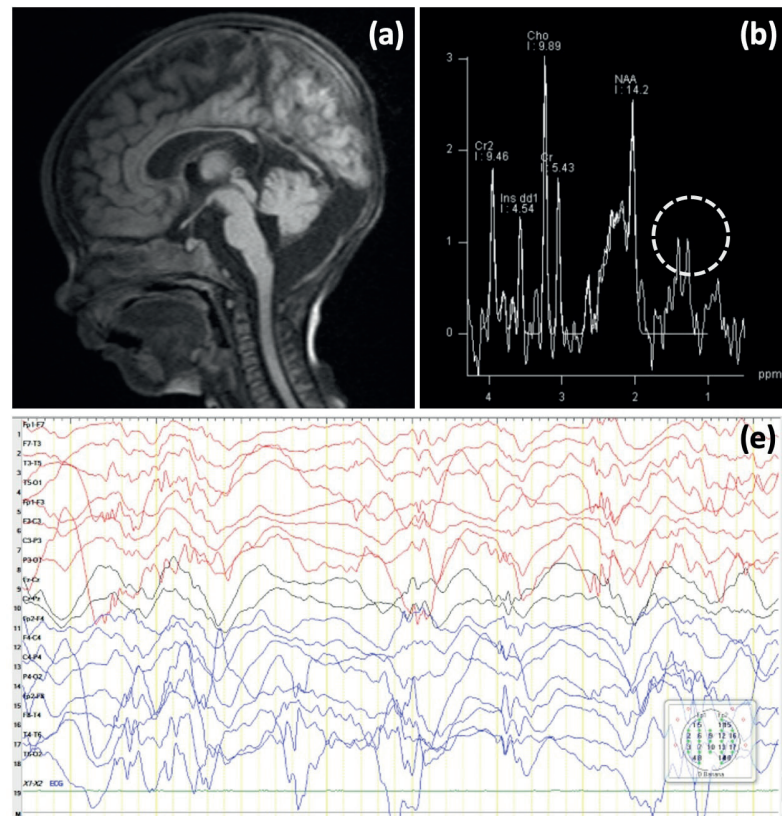

(b)

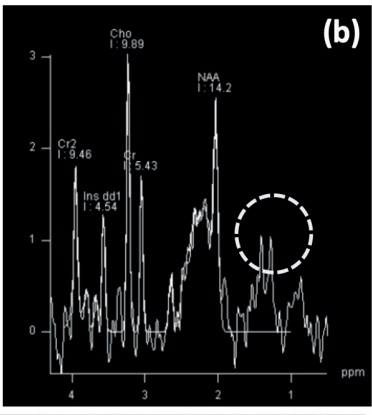

(e) (c)
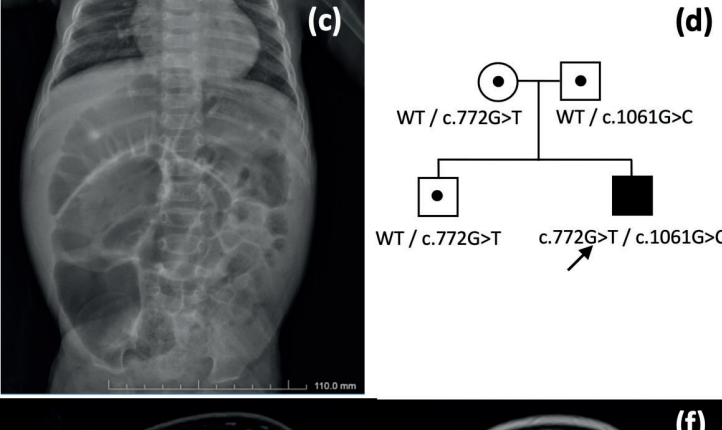

(d)
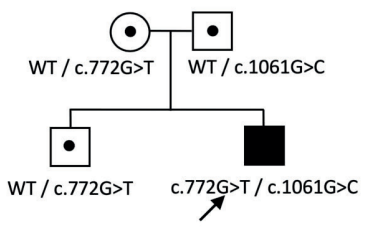

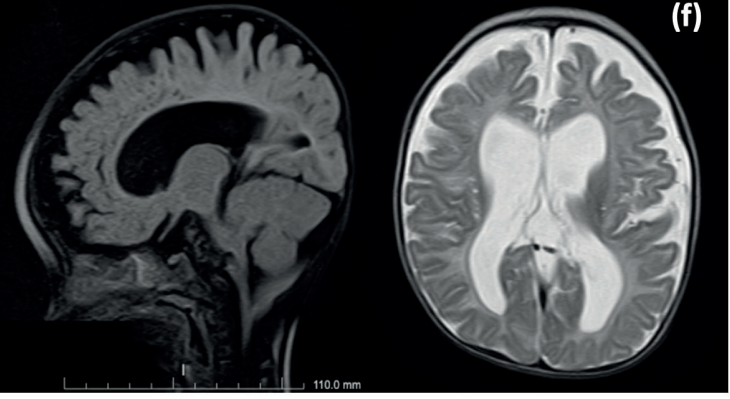

Fig. 2. (a) Thin corpus callosum in MRI. (b) Lactate pick on MRS. (c) Dilated intestinal loops. (d) Pedigree of the patient's family. (e) EEG findings of patient. (f) Enlarged ventricles, cerebral and cerebellar atrophy in MRI. 
At his most recent admission at 11 months of age, the patient was followed up with supportive treatments for developmental delay, seizures and eye involvement by a multidisciplinary team including specialists from pediatric metabolism, neurology, ophthalmology, nutrition and developmental pediatrics departments. Written informed consent was recieved from the family.

\section{Discussion}

To the best of our knowledge, our patient is the $95^{\text {th }}$ case of FBXL4-related MTDPS13 in the literature. ${ }^{4-7}$ We have identified two novel pathogenic variants in the FBXL-4 gene. While patients with FBXL4-related MTDPS13 presented with hypotonia, lactic acidosis and development delay in previous reports, our patient presented with dysmotility and lower gastrointestinal bleeding as an encephalomyopathic form of mitochondrial DNA depletion syndromes. Fukuyama et al. ${ }^{8}$ reported a patient with mitochondrial myopathy, encephalopathy, lactic acidosis, and stroke-like episodes (MELAS) which presented with transient non-occlusive small intestine ischemia that eventually triggered mucosal necrosis. They suggest that the presence of a large number of abnormal mitochondria in MELAS is closely linked to mucosal necrosis of the small intestine. Our patient was admitted with gastrointestinal bleeding as previously reported in literature. However, he didn't need any operation or intestinal resection and gastrointestinal bleeding spontaneously resolved. These findings were not compatible with intestinal ischemia. Furthermore, we did not perform endoscopy for the evaluation of gastrointestinal bleeding.

Typical manifestations of FBXL4-related MTDPS13, i.e. lactic acidosis, neurological involvement, developmental delay, feeding difficulties were also observed in our patient. However, microcephaly and hyperammonemia, which were observed in almost half of previous cases, were not noted in the patient presented herein. ${ }^{4}$ Cardiac and genitourinary (hypospadias, cryptorchidism) manifestations, renal tubular acidosis and hearing impairment -which are less frequent- were also not detected. ${ }^{2}$ We observed clinical findings such as epilepsy and nystagmus during the clinical follow-up which were not detected at the time of first admission. These findings suggest that longterm follow-up is required to better understand the clinical progress of the condition as well as the genotype-phenotype correlation.

Epilepsy is reported in $28 \%$ of affected patients, mainly in the form of generalized tonic-clonic seizures. Drug-resistant epilepsy has not been reported in any of the cases presented thus far. ${ }^{5}$ To date, ketogenic diet was initiated in a patient with FBXL4-related MTDPS13 due to differential diagnosis of pyruvate dehydrogenase complex deficiency which was then discontinued owing to elevated alanine aminotransferase and aspartate aminotransferase levels..$^{9}$ In our case, we initiated ketogenic diet due to intractable epilepsy; however, severe metabolic acidosis led us to interrupt the diet.

Abnormalities in brain MRI have been observed in almost all individuals with FBXL4-related MTDPS13. White matter abnormalities, cerebral atrophy and lactate peak in MRS are the most common findings in neuroimaging. Thin corpus callosum and cerebellar atrophy are detected in $20 \%$ of patients. ${ }^{2,410}$ Consistent with previous reports, in our case we determined enlarged ventricles due to cerebral atrophy together with thin corpus callosum and lactate peak in MRS. Cerebellar atrophy was noted during the clinical course.

Currently, there is no treatment for FBXL4related MTDPS13, and supportive treatment is essential. Administration of vitamins and antioxidants have limited benefits, similar to other mitochondrial disorders. ${ }^{2}$ In our experience, we have not detected any positive effects of the mitochondrial cocktail.

We identified two novel pathogenic variants, a stop-gain variant [p.Gly258* (c.772G $>$ T, Exon 5)] located in the F-box region and a missense 
variant [p.Trp354Ser (c.1061G>C, Exon 6)] located in leucin-rich repeats region of FBXL4. Although no genotype-phenotype correlation has been determined, El-Hattab et al. ${ }^{4}$ reported that patients with biallelic missense variants or compound heterozygous mutations of missense and null variants have longer survival compared to patients with biallelic null variants.

Huemer et al. ${ }^{10}$ and Gai et al. ${ }^{11}$ reported that mutations in the FBXL4 are associated with decreased respiratory chain complex activity in their functional studies on mitochondrial membrane potential. They also showed severe mtDNA depletion in patients with FBXL4 gene mutations. On the other hand, Barøy et al. ${ }^{9}$ found normal respiratory chain complex activity. It is important to assess both genetic findings and functional tests to better understand the disease and the effects of variants. However, we were not able to evaluate these parameters in our patient.

Our case suggests that patients with FBXL4-related MTDPS13 may present with gastrointestinal bleeding. This is the first report that shows intractable epilepsy in FBXL4related MTDPS13 and failure of ketogenic diet due to metabolic acidosis. Functional tests are required to reveal the unknowns of this disease.

\section{Acknowledgments}

This research did not receive any specific grant from funding agencies in the public, commercial, or not-for-profit sectors.

\section{REFERENCES}

1. Bonnen PE, Yarham JW, Besse A, et al. Mutations in FBXL4 cause mitochondrial encephalopathy and a disorder of mitochondrial DNA maintenance. Am J Hum Genet 2013; 93: 471-481.
2. Almannai M, Dai H, El-Hattab AW, Wong LJC. FBXL4-related encephalomyopathic mitochondrial DNA depletion syndrome. In: Adam MP, Ardinger $\mathrm{HH}$, Pagon RA, Wallace SE, Bean LJH, Stephens K, Amemiya A (eds). GeneReviews ${ }^{\circledR}$ [Internet]. Seattle (WA): University of Washington, Seattle, 2017. Available from http://www.ncbi.nlm.nih.gov/books/ NBK425540/.

3. Dai H, Zhang VW, El-Hattab AW, et al. FBXL4 defects are common in patients with congenital lactic acidemia and encephalomyopathic mitochondrial DNA depletion syndrome. Clin Genet 2017; 91: 634639.

4. El-Hattab AW, Dai H, Almannai M, et al. Molecular and clinical spectra of FBXL4 deficiency. Hum Mutat 2017; 38: 1649-1659.

5. Ballout RA, Al Alam C, Bonnen PE, Huemer M, ElHattab AW, Shbarou R. FBXL4-related mitochondrial DNA depletion syndrome 13 (MTDPS13): a case report with a comprehensive mutation review. Front Genet 2019; 10: 39.

6. Shamseldin HE, Alshammari M, Al-Sheddi T, et al. Genomic analysis of mitochondrial diseases in a consanguineous population reveals novel candidate disease genes. J Med Genet 2012; 49: 234-241.

7. Antoun G, McBride S, Vanstone JR, et al. Detailed biochemical and bioenergetic characterization of FBXL4-related encephalomyopathic mitochondrial DNA depletion. JIMD Rep 2016; 27: 1-9.

8. Fukuyama K, Ishikawa Y, Ogino T, et al. Mucosal necrosis of the small intestine in myopathy, encephalopathy, lactic acidosis, and stroke-like episodes syndrome. World J Gastroenterol 2012; 18: 5986-5989.

9. Barøy T, Pedurupillay CR, Bliksrud YT, et al. A novel mutation in FBXL4 in a Norwegian child with encephalomyopathic mitochondrial DNA depletion syndrome 13. Eur J Med Genet 2016; 59: 342-346.

10. Huemer M, Karall D, Schossig A, et al. Clinical, morphological, biochemical, imaging and outcome parameters in 21 individuals with mitochondrial maintenance defect related to FBXL4 mutations. J Inherit Metab Dis 2015; 38: 905-914.

11. Gai X, Ghezzi D, Johnson MA, et al. Mutations in FBXL4, encoding a mitochondrial protein, cause early-onset mitochondrial encephalomyopathy. Am J Hum Genet 2013; 93: 482-495. 